\title{
Stereotactic radiosurgery for spinal metastases: a literature review
}

\author{
Radiocirurgia estereotáxica para metástases de coluna vertebral: revisão de literatura
}

\author{
Andrei Fernandes Joaquim ${ }^{1}$, Enrico Ghizoni ${ }^{1}$, Helder Tedeschi ${ }^{1}$, Eduardo Baldon Pereira ${ }^{1}$, Leonardo Abdala Giacomini ${ }^{1}$
}

\begin{abstract}
Objective: The spine is the most common location for bone metastases. Since cure is not possible, local control and relief of symptoms is the basis for treatment, which is grounded on the use of conventional radiotherapy. Recently, spinal radiosurgery has been proposed for the local control of spinal metastases, whether as primary or salvage treatment. Consequently, we carried out a literature review in order to analyze the indications, efficacy, and safety of radiosurgery in the treatment of spinal metastases. Methods: We have reviewed the literature using the PubMed gateway with data from the MEDLINE library on studies related to the use of radiosurgery in treatment of bone metastases in spine. The studies were reviewed by all the authors and classified as to level of evidence, using the criterion defined by Wright. Results: The indications found for radiosurgery were primary control of epidural metastases (evidence level II), myeloma (level III), and metastases known to be poor responders to conventional radiotherapy - melanoma and renal cell carcinoma (level III). Spinal radiosurgery was also proposed for salvage treatment after conventional radiotherapy (level II). There is also some evidence as to the safety and efficacy of radiosurgery in cases of extramedullar and intramedullar intradural metastatic tumors (level III) and after spinal decompression and stabilization surgery. Conclusion: Radiosurgery can be used in primary or salvage treatment of spinal metastases, improving local disease control and patient symptoms. It should also be considered as initial treatment for radioresistant tumors, such as melanoma and renal cell carcinoma.
\end{abstract}

Keywords: Radiosurgery; Neoplasm metastasis/therapy; Spinal neoplasms/ radiotherapy

\section{RESUMO}

Objetivo: A coluna vertebral é o local mais comum de metástases ósseas. Uma vez que a cura não pode ser obtida, o controle local e 0 alívio dos sintomas é a base do tratamento, sendo este fundamentado no uso de radioterapia convencional. Recentemente, a radiocirurgia espinhal foi proposta para o controle local das metástases na coluna, seja como tratamento primário ou de resgate. Dessa forma, realizamos uma revisão da literatura para analisar as indicações, a eficácia e a segurança da radiocirurgia no tratamento das metástases da coluna. Métodos: A revisão de literatura foi realizada no portal PubMed dados da biblioteca MEDLINE, sobre os estudos relacionados ao uso da radiocirurgia no tratamento para metástases ósseas na coluna vertebral. Os estudos foram revisados por todos os autores e classificados quanto ao nível de evidência, utilizando critério definido por Wright. Resultados: As indicações encontradas para radiocirurgia foram: controle primário de metástases epidurais (nível II de evidência), mieloma (nível III) e metástases sabidamente pouco responsivas à radioterapia convencional - melanoma e carcinoma de células renais - (nível III). A radiocirurgia espinhal também foi proposta para o tratamento de resgate após falha da radioterapia convencional (nível II). Existe ainda alguma evidência quanto à segurança e a eficácia da radiocirurgia em casos de tumores metastáticos intradurais extramedulares e intramedulares (nível III), e após cirurgias de descompressão e estabilização da coluna. Conclusão: A radiocirurgia, portanto, pode ser usada no tratamento primário ou de resgate de metástases espinhais, melhorando o controle local da doença e dos sintomas dos pacientes. Deve ainda ser considerada como tratamento inicial para tumores radiorresistentes, como melanoma e carcinoma de células renais.

Descritores: Radiocirurgia; Metástase neoplásica/terapia; Neoplasias da coluna vertebral/radioterapia

\section{INTRODUCTION}

The spine is the most common site for bone metastases ${ }^{(1)}$. Spinal involvement occurs in up to $40 \%$ of patients with cancer during progression of the disease, with 5 to $10 \%$ of these patients developing epidural compression at some point of their progression ${ }^{(2)}$. More than $90 \%$ of the spinal metastases are located extradurally, whereas about $5 \%$

\footnotetext{
Universidade Estadual de Campinas, Campinas, SP, Brazil.

Corresponding author: Andrei Fernandes Joaquim - Rua Vital Brasil, 251 - Cidade Universitária Zeferino Vaz Campinas - Barão Geraldo - Zip code: 13083-888 - Campinas, SP, Brazil - Phone: (55 19) 3521 -7489 E-mail: andjoaquim@yahoo.com
}

Received on: Jan 6, 2013 - Accepted on: June 5, 2013 
are intradural and less than $1 \%$ are intramedullar ${ }^{(3)}$. Symptomatic compression occurs more frequently in the thoracic spine (50 to $70 \%$ ), followed by the lumbar spine (20 to $30 \%$ ) and the cervical spine (10 to $30 \%)^{(4)}$. This probably occurs because the thoracic canal has the smallest diameter and the largest number of vertebrae in the spine. The posterior half of the vertebral body is the most commonly involved ${ }^{(4)}$, spreading latterly to other regions (anterior body, lamina, and pedicles). Also important is the fact that by the time of diagnosis, multiple lesions can be found at noncontiguous levels in up to $40 \%$ of the patients. Regarding histology, up to $50 \%$ of the metastases come from one of the three following cancers: breast, lung, or prostate ${ }^{(1)}$.

Since spinal metastases suggest a non-controlled tumor, treatment is mainly based on palliation and local disease control. Although in selected cases chemotherapy can be used, radiotherapy, with or without concomitant surgery, remains the main treatment modality ${ }^{(5,6)}$. Surgical techniques have progressed substantially in the last years, allowing circumferential decompression of the spinal cord and spinal fixation for early stabilization $^{(6)}$. The main goals of surgery are spinal stabilization, restoration, and maintenance of the global alignment and decompression of neural structures, especially in radioresistant tumors ${ }^{(5)}$. Surgery can also be an option in unknown primary tumors (when a tissue sample is necessary) or in cases where local pain persists after other treatment modalities have been used. However, the surgical option should only be considered for patients with good performance scores, since it may entail serious morbidity and possible medical complications. Prognostic scoring systems, like the one proposed by Tokuhashi et al. and Tomita et al., are useful in surgical decision-making ${ }^{(7,8)}$.

\section{Role of radiation therapy in spinal metastases}

Since surgery is only considered in selected cases, conventional external beam radiotherapy (CER) remains the mainstay treatment modality for spinal metastases. The total dose of radiation ranges from 25 to $40 \mathrm{~Gy}$, fractionated daily into 8 to 20 doses $^{(6)}$. A broad margin is used in the radiation field, typically one or two vertebral segments above and two segments below the affected level in order to compensate for internal vertebral motion. This technique exposes healthy tissue to radiation, including the sensitive spinal cord. The dose of radiation is fractioned to allow recovery of the normal tissue, thus improving tolerance ${ }^{(6)}$.

Spinal stereotactic radiosurgery (SRS) is a more recently developed type of radiation therapy that delivers a high dose of radiation to the tumor, whilst minimizing the amount delivered to the healthy neighboring tissues. The target is defined by high-resolution stereotactic imaging, and requires rigid spine immobilization to offer a precise conformal dose, using frame or frameless techniques, based on external immobilization devices with fiducial markers. Treatment is fractionated into fewer sessions (one to five), with total doses ranging from 8 to $30 \mathrm{~Gy}$. When one session is used, the term "radiosurgery" must be preferentially used, whereas "fractionated stereotactic radiosurgery" is better used when multiple sessions are performed. Early versions of SRS in the spine required surgical fixation of frames in the spinous processes to avoid movement artifacts. The novel systems are frameless, based on internal skeletal anatomy, and implanted fiducial points, or infrared surface markers, with near real-time images for motion correction. Radiation intensity modulation increases the conformity of radiation to the tumor, minimizing the dose to normal tissue ${ }^{(6)}$. However, the effectiveness, safety, precise indications, and long-term evaluation regarding disease control of the effects of SRS remain unclear.

\section{OBJECTIVE}

To perform a literature review of the indications, safety, and efficacy of spinal radiosurgery when compared to conventional external radiotherapy for the treatment of spinal metastases.

\section{METHODS}

\section{Search strategy}

A literature review using the PubMed gateway of the MEDLINE database was performed, with no time restriction, until September, 2012. The following key-words were queried, singly or in combination: "radiosurgery", "stereotactic", "spinal metastases", "spinal cancer", "treatment", "indications", "radiation therapy", and "extracranial".

\section{Study selection}

We included clinical papers of patients who received radiosurgery for the treatment of spinal metastases.

We excluded non-English language papers, literature reviews, case reports, and papers not focused on radiosurgical treatment of spinal metastases.

\section{Data extraction}

One hundred and twenty-four studies were reviewed and scrutinized according to study design (retrospective 
case series, prospective studies, and randomized control trials), patient characteristics, and primary findings by four independent researchers (AFJ, EG, HT, LG). After analysis of the four authors, reselection was made and a total of 31 studies were selected and included in our review.

\section{Measurements and outcome evaluation}

The results of the studies were grouped according to their main purpose: pain control, radiological control, overall survival, comparison of treatment modalities (SRS versus CER versus surgery), indications for spinal radiosurgery, and methods of evaluating disease control and response to treatment.

\section{Evaluation of the level of evidence}

The studies were classified according to the level of evidence for therapeutic studies, defined by Wright et al. ${ }^{(9)}$.

1. Higher quality randomized controlled trial.

2. Lesser quality randomized controlled trial; prospective comparative study.

3. Case-control study; retrospective comparative study.

4. Case series.

5. Expert opinion.

\section{RESULTS}

Out of 31 selected studies, a total of 2,241 patients were treated for spinal metastases. All papers are summarized on chart 1.

Chart 1. Selected studies after a systematic review using the MEDLINE database

\begin{tabular}{|c|c|c|c|c|c|}
\hline & Study type & $\begin{array}{c}\text { Radiosurgery } \\
\text { status/indication }\end{array}$ & $\mathbf{n}$ & Dose & Results \\
\hline Ahmed et al. ${ }^{(10)}$ & $\begin{array}{l}\text { Prospective } \\
\text { case series }\end{array}$ & $\begin{array}{l}\text { Primary and } \\
\text { reirradiation therapy for } \\
\text { spinal metastases }\end{array}$ & $\begin{array}{l}66 \text { patients (85 } \\
\text { lesions) }\end{array}$ & $\begin{array}{l}\text { 24Gy (10-40Gy) } \\
\text { (median } 3 \text { fractions, } \\
\text { range to } 1-5)\end{array}$ & $\begin{array}{l}\text { The mean actuarial survival at } 12 \text { months was } 52.2 \% \\
7 \text { patients had both local and marginal failure, } 1 \text { patient experienced } \\
\text { marginal but not local failure, and } 1 \text { patient had local failure only } \\
\text { Actuarial local control at } 1 \text { year was } 83.3 \% \text { and } 91.2 \% \text { in patients with } \\
\text { and without prior RT, respectively } \\
\text { No Grade } 4 \text { toxicities were reported }\end{array}$ \\
\hline Amdur et al. ${ }^{(11)}$ & $\begin{array}{l}\text { Prospective } \\
\text { case series }\end{array}$ & $\begin{array}{l}\text { Primary and } \\
\text { reirradiation therapy for } \\
\text { spinal metastases }\end{array}$ & $\begin{array}{l}21 \text { patients }(9 \\
\text { patients; no prior } \\
\text { radiotherapy and } 12 \\
\text { patients with prior } \\
\text { radiotherapy) }\end{array}$ & $\begin{array}{l}\text { 15Gy (no prior spinal } \\
\text { radiotherapy) } \\
\text { 5Gy (prior spinal } \\
\text { radiotherapy) }\end{array}$ & $\begin{array}{l}\text { No late toxicities } \\
3 \text { patients experienced radiographic evidence of vertebral body } \\
\text { compression in field } \\
43 \% \text { experienced pain relief } \\
1 \text {-year progression-free survival was } 5 \% \text { with } 60 \% \text { of patients dead } \\
\text { by } 1 \text { year } \\
\text { In patients with and without prior radiotherapy, they achieved the } \\
\text { target-coverage goal in } 91 \% \text { and } 95 \% \text {, respectively }\end{array}$ \\
\hline Benzil et al. ${ }^{(12)}$ & Case series & $\begin{array}{l}\text { Primary and } \\
\text { reirradiation therapy for } \\
\text { spinal metastases and } \\
\text { primary tumors }\end{array}$ & $\begin{array}{l}31 \text { patients (35 } \\
\text { lesions); } 26 \\
\text { metastases and } \\
4 \text { intradural and } 5 \\
\text { extradural tumors }\end{array}$ & $\begin{array}{l}\text { Mean single dose } \\
\text { 2.68Gy and mean } \\
\text { total dose } 6.89 \mathrm{~Gy} \text { for } \\
\text { intradural tumors }\end{array}$ & $\begin{array}{l}\text { Significant pain relief was achieved in } 32 / 34 \text { treated tumors } \\
\text { Pain relief was achieved with a single dose as low as } 5 \mathrm{~Gy} \\
2 \text { patients experienced transient radiculitis (both with a BED > 60Gy) } \\
\text { No patient experienced other organ toxicity }\end{array}$ \\
\hline Chang et al. ${ }^{(13)}$ & Case series & $\begin{array}{l}\text { Primary radiosurgery } \\
\text { for spinal metastases }\end{array}$ & 63 patients & $\begin{array}{l}\text { 27-30Gy (three } 9 G y \\
\text { fractions and five } 6 \mathrm{~Gy} \\
\text { fractions) }\end{array}$ & $\begin{array}{l}\text { No neurological complications (median of } 21.3 \text { months of follow-up) } \\
\text { 1-year tumor progression-free was } 84 \% \\
\text { Mild symptoms of toxicity (nausea, vomiting, and diarrhea) }\end{array}$ \\
\hline Chang et al..$^{(14)}$ & Case series & $\begin{array}{l}\text { Primary and } \\
\text { reirradiation therapy for } \\
\text { spinal metastases }\end{array}$ & $\begin{array}{l}129 \text { patients (53 } \\
\text { reirradiation) }\end{array}$ & $\begin{array}{l}\text { 16-39Gy in 1-5 } \\
\text { fractions }\end{array}$ & $\begin{array}{l}\text { Pain relief in } 91 \% \\
\text { In } 108 \text { lesions, } 75 \text { decreased or stable mass size }\end{array}$ \\
\hline Chang et al. (15) & Case series & $\begin{array}{l}\text { Primary and } \\
\text { reirradiation therapy for } \\
\text { spinal metastases }\end{array}$ & $\begin{array}{l}54 \text { retreatment and } \\
131 \text { initial SBRT }\end{array}$ & $\begin{array}{l}\text { Mean radiation } \\
\text { doses to tumor } \\
\text { margin } 51.1 \mathrm{~Gy} 2 / 10 \\
\text { (retreatment) and } \\
50.7 \mathrm{~Gy} 2 / 10 \text { (initial } \\
\text { treatment) }\end{array}$ & $\begin{array}{l}\text { Mean progression-free period was } 23.9 \text { months (overall); } 18 \text { months } \\
\text { (retreatment) and } 26 \text { months (initial treatment) } \\
\text { Radiological control rates were about } 95 \% \text { at } 6 \text { months and up to } \\
80 \% \text { at } 12 \text { months } \\
\text { No radiation myelopathy }\end{array}$ \\
\hline De Salles et al. ${ }^{(16)}$ & Case series & $\begin{array}{l}\text { Radiosurgery for spinal } \\
\text { metastases and benign } \\
\text { spinal tumors }\end{array}$ & $\begin{array}{l}14 \text { patients (22 } \\
\text { lesions); } 11 \text { patients } \\
\text { with metastases, } 2 \\
\text { neurofibromas, and } 1 \\
\text { meningioma }\end{array}$ & $\begin{array}{l}\text { A mean dose of } \\
12 \pm 2.7 \mathrm{~Gy} \text { (range } \\
8-21 \mathrm{~Gy}) \\
\times 13 \text { received single- } \\
\text { dose stereotactic } \\
\text { radiosurgery }\end{array}$ & $\begin{array}{l}\text { Mean follow-up period was } 6.1 \pm 3.9 \text { months (range } 1-16 \text { months) } \\
3 \text { patients became pain-free and } 4 \text { experienced considerable relief } \\
\text { Weakness improved in } 2 \text { patients with this preoperative symptom and } \\
\text { the asymptomatic patients remained asymptomatic } \\
4 \text { lesions decreased in size, } 5 \text { remained stable, } 7 \text { progressed, and } 6 \\
\text { were not followed ( } 2 \text { patients died before follow-up) } \\
4 \text { patients in all died, } 3 \text { of systemic disease and } 1 \text { of thoracic lesion } \\
\text { progression } \\
\text { No complications were observed }\end{array}$ \\
\hline
\end{tabular}


...continuation

Chart 1. Selected studies after a systematic review using the MEDLINE database

\begin{tabular}{|c|c|c|c|c|c|}
\hline & Study type & $\begin{array}{c}\text { Radiosurgery } \\
\text { status/indication }\end{array}$ & $\mathbf{n}$ & Dose & Results \\
\hline Garg et al. ${ }^{(17)}$ & $\begin{array}{l}\text { Prospective } \\
\text { case series }\end{array}$ & $\begin{array}{l}\text { Primary radiosurgery } \\
\text { for spinal metastases }\end{array}$ & $\begin{array}{l}61 \text { patients (63 } \\
\text { tumors) }\end{array}$ & $\begin{array}{c}\text { 16-24Gy } \\
\text { (single fraction) }\end{array}$ & $\begin{array}{l}\text { Mean follow-up of } 20 \text { months } \\
\text { Actuarial 18-month imaging local control rate for all patients was } 88 \% \\
\text { Actuarial 18-month overall survival rate for all patients was } 64 \% \text { with a } \\
\text { median survival for all patients of } 30 \text { months } \\
\text { No significant differences in outcomes were noted with respect to tumor } \\
\text { histology or dose } \\
\text { The data support an expanded indication for spinal radiosurgery as first- } \\
\text { line treatment }\end{array}$ \\
\hline Levine et al. ${ }^{(18)}$ & Case series & $\begin{array}{l}\text { Primary radiosurgery } \\
\text { for primary spinal } \\
\text { sarcomas and } \\
\text { metastases }\end{array}$ & 24 patients & 30Gy & $\begin{array}{l}7 \text { patients definitively treated; } 2 \text { complete regression, } 3 \text { partial regression, } \\
\text { and } 2 \text { late recurrence re-treated } \\
7 \text { had surgery }+ \text { SRS; } 5 \text { cases of total tumor control with mean follow-up } \\
\text { of } 43.5 \text { months } \\
10 \text { patients with metastases; all died; mean } 11.1 \text { months of survival; } \\
80 \% \text { pain relief } \\
\text { No myelitis }\end{array}$ \\
\hline Martin et al. ${ }^{(19)}$ & Case series & $\begin{array}{l}\text { Primary (14 lesions) or } \\
\text { metastatic (39) spinal } \\
\text { lesions }\end{array}$ & $\begin{array}{l}41 \text { patients (53 } \\
\text { lesions) }\end{array}$ & $\begin{array}{c}\text { 8-30Gy } \\
\text { (1-3 fractions) }\end{array}$ & $\begin{array}{l}\text { Median follow-up of } 11.1 \text { months } \\
59 \% \text { of patients experienced no acute side effects from treatment; there } \\
\text { were } 3 \text { cases of acute grade } 3 \text { toxicity } \\
\text { Local control and overall survival were } 91 \text { and } 65 \% \text {, respectively } \\
\text { Pain improvement was seen in } 75 \% \text { of symptomatic metastases at } 6 \\
\text { months post-treatment }\end{array}$ \\
\hline Patel et al. ${ }^{(20)}$ & Case series & $\begin{array}{l}\text { Primary irradiation of } \\
\text { spinal metastases }\end{array}$ & $\begin{array}{c}117 \text { patients (154 } \\
\text { lesions) }\end{array}$ & $\begin{array}{l}\text { Single fraction } \\
\text { comparing whole } \\
\text { (W) versus partial (P) } \\
\text { contour approach of } \\
\text { the vertebral body }\end{array}$ & $\begin{array}{l}\text { W Group had a lower re-treatment rate ( } 11 \% \text { for W Group versus } 18.6 \% \\
\text { for P Group; } p=0.285) \\
\text { Prior surgery status ( } \beta=1.953 ; O R=7.052 ; p<0.001) \text { was correlated to } \\
\text { the re-treatment rate } \\
\text { The } 2 \text {-year survival was } 25.7 \% \text { in W Group and } 20.9 \% \text { in P Group ( } p=0.741) \\
\text { They concluded that contouring the whole vertebral body for stereotactic } \\
\text { body radiation therapy treatment of metastatic spinal lesions shows } \\
\text { potential benefits by reducing the risk of recurrence, improving } \\
\text { symptomatic relief, and providing improved local tumor control }\end{array}$ \\
\hline Ryu et al. ${ }^{\{21\}}$ & Case series & $\begin{array}{l}\text { Primary radiosurgery } \\
\text { for spinal metastases }\end{array}$ & $\begin{array}{l}49 \text { patients (61 } \\
\text { lesions) }\end{array}$ & $\begin{array}{c}\text { 10-16Gy } \\
\text { (single dose) }\end{array}$ & $\begin{array}{l}\text { Complete/partial relief in } 85 \% \\
\text { Relapse of pain in } 7 \% \\
5 \% \text { of radiologically adjacent spine metastases }\end{array}$ \\
\hline Wang et al.(22) & Case series & $\begin{array}{l}\text { Primary irradiation of } \\
\text { spinal metastases }\end{array}$ & $\begin{array}{l}149 \text { patients (166 } \\
\text { lesions) }\end{array}$ & $\begin{array}{l}\text { 27-30Gy } \\
\text { (3 doses) }\end{array}$ & $\begin{array}{l}\text { Median follow-up of } 15.9 \text { months } \\
\text { Pain control improved from } 26-54 \% \text { at } 6 \text { months } \\
\text { Progression-free survival after SBRT was } 80.5 \% \text { at } 1 \text { year }\end{array}$ \\
\hline Gerszten et al.(23) & Case series & $\begin{array}{c}\text { Primary and } \\
\text { reirradiation therapy } \\
\text { for renal cell carcinoma } \\
\text { metastases }\end{array}$ & $\begin{array}{l}48 \text { patients with } 60 \\
\text { RCC metastases } \\
\text { (42 lesions were } \\
\text { reirradiated) }\end{array}$ & $\begin{array}{c}\text { Tumor dose range } \\
\text { to } 17.5-25 \mathrm{~Gy} \\
\text { (mean } 20 \mathrm{~Gy}) \text {; } \\
\text { single dose }\end{array}$ & $\begin{array}{l}\text { Follow-up for } 14-48 \text { months (median } 37 \text { months) } \\
\text { No radiation-induced toxicity occurred during the follow-up period } \\
\text { Axial and radicular pain improved in } 34(89 \%) \text { of } 38 \text { patients who were } \\
\text { treated primarily for pain } \\
\text { Tumor control was demonstrated in } 7 \text { of } 8 \text { patients treated primarily for } \\
\text { radiographically documented tumor progression } \\
6 \text { patients required open surgical intervention for tumor progression that } \\
\text { had caused neurological dysfunction after radiosurgery }\end{array}$ \\
\hline Gerszten et al. ${ }^{(24)}$ & Case series & $\begin{array}{c}\text { Primary and } \\
\text { reirradiation therapy } \\
\text { for melanoma spinal } \\
\text { metastases }\end{array}$ & $\begin{array}{l}28 \text { patients ( } 36 \\
\text { lesions in } 23 \text { patients } \\
\text { with previous } \\
\text { external beam } \\
\text { irradiation) }\end{array}$ & $\begin{array}{l}\text { Maximum dose } \\
\text { was } 17.5-25 \mathrm{~Gy} \\
\text { (mean } 21.7 \mathrm{~Gy} \text { ); } \\
\text { single fraction }\end{array}$ & $\begin{array}{l}\text { Follow-up period of } 3-43 \text { months (median } 13 \text { months) } \\
\text { No radiation-induced toxicity occurred during the follow-up period } \\
\text { Axial and radicular pain improved in } 27 \text { of } 28 \text { patients ( } 96 \% \text { ) who were } \\
\text { treated primarily for pain } \\
\text { Long-term tumor control was seen in } 3 \text { of } 4 \text { cases treated primarily for } \\
+ \text { tumor progression. Two patients went on to require open surgical } \\
\text { intervention for tumor progression resulting in neurological deficit }\end{array}$ \\
\hline Choi et al. ${ }^{(25)}$ & Case series & $\begin{array}{l}\text { Reirradiation therapy } \\
\text { for spinal metastases } \\
\text { (median previous spinal } \\
\text { cord dose of } 40 \mathrm{~Gy} \text { ) }\end{array}$ & $\begin{array}{l}42 \text { patients (51 } \\
\text { lesions) }\end{array}$ & $\begin{array}{l}\text { Median dose of } \\
20 \mathrm{~Gy} \\
\text { (range } 10-30 \mathrm{~Gy} \text { ) in } \\
\text { 1-5 fractions } \\
\text { (median 2) }\end{array}$ & $\begin{array}{l}\text { Median follow-up of } 7 \text { months (range: } 2-47 \text { months) } \\
\text { The Kaplan-Meier local control and overall survival rates at } 6 / 12 \text { months } \\
\text { were } 87 \% / 73 \% \text { and } 81 \% / 68 \% \text {, respectively } \\
\text { Time to re-treatment of } \leq 12 \text { months and the combination of time to re- } \\
\text { treatment of } \leq 12 \text { months with an SSED of }<15 \mathrm{~Gy}(10) \text { were significant } \\
\text { predictors of local failure on univariate and multivariate analyses } \\
1 \text { patient }(2 \%) \text { experienced Grade } 4 \text { neurotoxicity }\end{array}$ \\
\hline
\end{tabular}


...continuation

Chart 1. Selected studies after a systematic review using the MEDLINE database

\begin{tabular}{|c|c|c|c|c|c|}
\hline & Study type & $\begin{array}{c}\text { Radiosurgery } \\
\text { status/indication }\end{array}$ & $\mathbf{n}$ & Dose & Results \\
\hline Gagnon et al.(26) & $\begin{array}{l}\text { Case series } \\
\text { (case control } \\
\text { study) }\end{array}$ & $\begin{array}{c}\text { Reirradiation therapy } \\
\text { for breast cancer spinal } \\
\text { metastases }\end{array}$ & $\begin{array}{l}18 \text { patients treated - } \\
\text { recurrent metastases } \\
\text { (reirradiated) } \\
\text { compared with } \\
18 \text { who received } \\
\text { conventional RDP }\end{array}$ & $\begin{array}{l}\text { Doses ranging } \\
\text { from } 21-28 \mathrm{~Gy} \\
\text { (3-5 fractions) }\end{array}$ & $\begin{array}{l}\text { Both groups were comparable along all matching dimensions and in } \\
\text { performance status before treatment } \\
\text { Outcomes of treatment were similar for patients in both groups; } \\
\text { ambulation, performance status, and pain worsened similarly across } \\
\text { groups post-treatment } \\
\text { Survival and the number of complications appeared to favor the } \\
\text { CyberKnife group, but the differences did not reach statistical } \\
\text { significance } \\
\text { Salvage CyberKnife is efficacious }\end{array}$ \\
\hline Klish et al. ${ }^{(27)}$ & $\begin{array}{l}\text { Prospective } \\
\text { case series }\end{array}$ & $\begin{array}{l}\text { Reirradiation for } \\
\text { previously irradiated } \\
\text { spinal metastases }\end{array}$ & 58 patients & $\begin{array}{l}\text { Adjacent level disease } \\
\text { after irradiation of the } \\
\text { involved vertebral body }\end{array}$ & $\begin{array}{l}\text { Avoid the historic irradiation of } 1-2 \text { vertebral bodies above/below the } \\
\text { involvement using EBRT } \\
\text { Multiple level of SRS irradiation is unnecessary } \\
3 \% \text { of failure compared with }<5 \% \text { of isolated failures of the } \\
\text { unirradiated adjacent vertebral body }\end{array}$ \\
\hline Koyfman et al. ${ }^{(28)}$ & Case series & $\begin{array}{l}\text { Reirradiation for } \\
\text { previously irradiated } \\
\text { spinal metastases }\end{array}$ & $\begin{array}{c}149 \text { patients (208 } \\
\text { lesions) }\end{array}$ & $\begin{array}{c}\text { 14Gy } \\
\text { (median dose } \\
\text { ranging from 10-16Gy) }\end{array}$ & $\begin{array}{l}\text { Median follow-up was } 8.6 \text { months, and median survival was } 12.8 \text { months } \\
\text { Recurrence occurred in } 26(12.5 \%) \text { treated lesions, at a median time } \\
\text { of } 7.7 \text { months after conventional radiotherapy } \\
\text { Patients with paraspinal disease at the time of conventional } \\
\text { radiotherapy }(20.8 \% \text { versus } 7.6 \% \text { of patients; } p=0.02) \text {, and those } \\
\text { treated with }<16 \mathrm{~Gy}(16.3 \% \text { versus } 6.3 \% \text { of patients; } p=0.14) \text { had } \\
\text { higher rates of recurrence }\end{array}$ \\
\hline Mahadevan et al..(29) & Case series & $\begin{array}{l}\text { Reirradiation for } \\
\text { previously irradiated } \\
\text { spinal metastases }\end{array}$ & 60 patients & $\begin{array}{c}8 \mathrm{~Gy} \times 3=24 \mathrm{~Gy} \\
\text { (far from the cord) } \\
5 \text { to } 6 \mathrm{~Gy} \times 5=25 \text { to } 30 \mathrm{~Gy} \\
\text { (near the cord) }\end{array}$ & $\begin{array}{l}9 \text { months median progression-free survival } \\
93 \% \text { had stability } \\
65 \% \text { had pain relief } \\
7 \% \text { disease progression } \\
\text { No toxicity }\end{array}$ \\
\hline Nikolajek et al.(30) & Case series & $\begin{array}{l}\text { Reirradiation for } \\
\text { previously irradiated } \\
\text { spinal metastases }\end{array}$ & $\begin{array}{c}54 \text { patients (70 } \\
\text { lesions) }\end{array}$ & $\begin{array}{l}\text { Median radiosurgery } \\
\text { dose: } 1 \times 18 \mathrm{~Gy} \\
\text { (range } 10-28 \mathrm{~Gy} \text { ) to the } \\
\text { median } 70 \% \text { isodose } \\
\quad \text { single fraction }\end{array}$ & $\begin{array}{l}\text { Median follow-up of } 14.5 \text { months } \\
\text { The actuarial rates of freedom from local failure at 6/12/18 months } \\
\text { were } 93 \%, 88 \% \text {, and } 85 \% \text {, respectively } \\
\text { In } 6 \text { out of } 7 \text { patients worse sensory or motor deficit after SRS was } \\
\text { caused by local or distant failures (diagnosed by CT/MRI) } \\
1 \text { patient with metastatic renal cell carcinoma developed a progressive } \\
\text { complete paraparesis } 1 \text { year after the last treatment at lumbar level L3 }\end{array}$ \\
\hline Sahgal et al.(31) & Case series & $\begin{array}{l}\text { Primary and } \\
\text { reirradiation for } \\
\text { previously irradiated } \\
\text { spinal metastases }\end{array}$ & $\begin{array}{l}39 \text { patients (60 } \\
\text { lesions) } \\
23 \text { lesions were } \\
\text { unirradiated } \\
37 \text { were reirradiated } \\
\text { (31 had image tumor } \\
\text { progression) }\end{array}$ & $\begin{array}{l}\text { Median total dose } \\
\text { prescribed was 24Gy } \\
\text { in } 3 \text { fractions }\end{array}$ & $\begin{array}{l}\text { Median survival time measured was } 21 \text { months ( } 95 \% \mathrm{Cl}=8-27 \text { months). } \\
\text { 2-year survival probability was } 45 \% \\
\text { Median tumor follow-up for the unirradiated and reirradiated group } \\
\text { was } 9 \text { months (range: } 1-26 \text { ) and } 7 \text { months (range: } 1-48 \text { ) respectively } \\
8 \text { of } 60 \text { tumors have progressed, and the } 1 \text { - and } 2 \text {-year PFP was } 85 \% \\
\text { and } 69 \% \text {, respectively } \\
\text { For the salvage group the } 1 \text {-year PFP was } 96 \% \\
\text { In } 6 \text { of } 8 \text { failures the minimum distance from the tumor to the thecal } \\
\text { sac was }<\text { or }=1 \text { mm } \\
39 / 60 \text { had >or }=6 \text { months follow-up and no radiation-induced myelopathy } \\
\text { or radiculopathy occurred }\end{array}$ \\
\hline Sahgal et al. ${ }^{(32)}$ & Case series & $\begin{array}{l}\text { Reirradiation for } \\
\text { previously irradiated } \\
\text { with external beam } \\
\quad \text { radiation }\end{array}$ & $\begin{array}{l}19 \text { patients ( } 5 \text { with } \\
\text { radiation myelopathy } \\
\text { - } \mathrm{RMI} \text { and } 14 \text { without } \\
\text { it - no-RMI) }\end{array}$ & $\begin{array}{l}\text { Mean of } 20 \mathrm{~Gy} \text { in the } \\
\text { no-RMl group versus } \\
1-5 \text { fractions } 67.4 \\
\text { in the RMI group }\end{array}$ & $\begin{array}{l}\text { SBRT given at least } 5 \text { months after conventional palliative radiotherapy } \\
\text { with a reirradiation dose of } 20-25 \mathrm{~Gy}(2 / 2) \text { appeared to be safe, } \\
\text { provided the total dose does not exceed approximately } 70 \mathrm{~Gy}(2 / 2)\end{array}$ \\
\hline Sheehan et al. ${ }^{(33)}$ & Case series & $\begin{array}{l}\text { Spinal radiosurgery } \\
\text { using a helical } \\
\text { Tomotherapy }\end{array}$ & $\begin{array}{l}40 \text { patients }(110 \\
\text { tumors); range } 1-6 \\
\text { tumors per patient } \\
23(57.5 \% \text { underwent } \\
\text { previous surgery }\end{array}$ & $\begin{array}{l}\text { Mean radiosurgical } \\
\text { dose was } 17.3 \mathrm{~Gy} \\
\text { (range: } 10-24 \mathrm{~Gy} \text { ) }\end{array}$ & $\begin{array}{l}\text { Mean follow-up duration of } 12.7 \text { months (range: } 4-32 \text { months) } \\
\text { Decreased or stable tumor volume was seen in } 90(82 \%) \text { of the } \\
\text { tumors treated } \\
\text { Pain improvement in } 34 \text { patients }(85 \%)\end{array}$ \\
\hline Shin et al. ${ }^{(34)}$ & Case series & $\begin{array}{l}\text { Intradural extramedullar } \\
\text { and intramedullar } \\
\text { spinal metastases }\end{array}$ & 9 patients & $\begin{array}{c}\text { 13.8Gy } \\
\text { (10-16Gy) }\end{array}$ & $\begin{array}{l}80 \% \text { improved symptoms } \\
10 \% \text { worsened } \\
\text { No radiation toxicity }\end{array}$ \\
\hline Jin et al..$^{(35)}$ & $\begin{array}{l}\text { Retrospective } \\
\text { case series }\end{array}$ & $\begin{array}{l}\text { Radiosurgery for } \\
\text { epidural myeloma }\end{array}$ & 24 patients & $\begin{array}{c}\text { 10-18Gy } \\
\text { (16Gy) }\end{array}$ & $\begin{array}{l}81 \% \text { had complete radiographic response } \\
86 \% \text { pain control } \\
71 \% \text { of improvement in neurological symptoms }\end{array}$ \\
\hline
\end{tabular}


continuation

Chart 1. Selected studies after a systematic review using the MEDLINE database

\begin{tabular}{|c|c|c|c|c|c|}
\hline & Study type & $\begin{array}{c}\text { Radiosurgery } \\
\text { status/indication }\end{array}$ & $\mathbf{n}$ & Dose & Results \\
\hline Massicote et al. ${ }^{(36)}$ & Case series & $\begin{array}{l}\text { Primary irradiation } \\
\text { after minimally } \\
\text { invasive spinal surgery } \\
\text { for unstable spinal } \\
\text { metastases }\end{array}$ & 10 patients & & $\begin{array}{l}8 \text { patients were symptomatic at baseline } \\
\text { The median follow-up was } 13 \text { months (range: } 3-18 \text { ) } \\
\text { Following surgery, the median time to SBRT treatment } \\
\text { planning was } 6.5 \text { days and subsequent median time to } \\
\text { treatment was } 7 \text { days } \\
\text { Local control was observed in } 7 \text { of the } 10 \text { patients. } \\
\text { Improvements in VAS, ODI, and OOL were observed post- } \\
\text { SBRT }\end{array}$ \\
\hline Moulding et al..$^{(37)}$ & Case series & $\begin{array}{l}\text { Surgical } \\
\text { decompression and } \\
\text { stabilization for epidural } \\
\text { compression followed } \\
\text { by spinal radiosurgery }\end{array}$ & $\begin{array}{l}21 \text { patients } \\
\text { (20 tumors; } 95 \% \text { were } \\
\text { considered highly } \\
\text { radioresistant to } \\
\text { conventional external } \\
\text { beam radiotherapy) }\end{array}$ & $\begin{array}{l}\text { 18-24Gy (median } 24) \\
\text { single dose } \\
\text { Planned target volume } \\
\text { received a high dose } \\
(24 \mathrm{~Gy}) \text { in } 16 \text { patients } \\
(76.2 \%) \text {, and a low } \\
\text { dose }(18 \text { or } 21 \mathrm{~Gy}) \text { in } \\
5 \text { patients }(23.8 \%)\end{array}$ & $\begin{array}{l}15(72 \%) \text { of } 21 \text { patients died, and in all cases death was due to } \\
\text { systemic progression as opposed to local failure } \\
\text { The median overall survival after radiosurgery was } 310 \text { days } \\
\text { One patient }(4.8 \%) \text { underwent repeat surgery for local failure } \\
\text { and } 2 \text { patients }(9.5 \%) \text { underwent spine surgery for other } \\
\text { reasons } \\
\text { Local control was maintained after radiosurgery in } 17 \text { (81\%) } \\
\text { of } 21 \text { patients until death or most recent follow-up, with an } \\
\text { estimated 1-year local failure risk of } 9.5 \% \\
\text { Of the failures, } 3 \text { of } 4 \text { were noted in patients receiving low- } \\
\text { dose radiosurgery } \\
\text { Patients receiving adjuvant stereotactic radiosurgery with } \\
\text { a high dose had a } 93.8 \% \text { overall local control rate (15 of } 16 \\
\text { patients), with a 1-year estimated failure risk of } 6.3 \%\end{array}$ \\
\hline Garg et al. ${ }^{(38)}$ & $\begin{array}{c}\text { Prospective case } \\
\text { series }\end{array}$ & $\begin{array}{l}\text { Reirradiation therapy } \\
\text { for spinal metastases }\end{array}$ & $\begin{array}{l}59 \text { patients } \\
\text { (63 tumors) }\end{array}$ & $\begin{array}{l}\text { 30Gy (5 fractions; 6Gy) } \\
27 \mathrm{~Gy} \text { (3 fractions; 9Gy) }\end{array}$ & $\begin{array}{l}\text { 1-year overall survival in } 76 \% \text { with local control } \\
92 \% \text { freedom from neurological injury } \\
81 \% \text { of the tumors within } 5 \mathrm{~mm} \text { of the spinal cord developed } \\
\text { cord compression }\end{array}$ \\
\hline Gerszten et al. ${ }^{(39)}$ & $\begin{array}{l}\text { Prospective } \\
\text { nonrandomized } \\
\text { cohort study }\end{array}$ & $\begin{array}{c}\text { Primary and } \\
\text { reirradiation therapy } \\
\text { for spinal metastases }\end{array}$ & 500 cases & $\begin{array}{l}\text { Maximum intratumoral } \\
\text { dose range from } \\
\text { 12.5-25Gy (mean } 20 \mathrm{~Gy} \text { ) } \\
\text { (single fraction) }\end{array}$ & $\begin{array}{l}\text { Long-term pain improvement occurred in } 290 \text { of } 336 \text { cases } \\
(86 \%) \\
\text { Long-term tumor control was demonstrated in } 90 \% \text { of lesions } \\
\text { treated with radiosurgery as a primary treatment modality and } \\
\text { in } 88 \% \text { of lesions treated for radiographic tumor progression } \\
27 \text { of } 32 \text { cases ( } 84 \%) \text { with a progressive neurologic deficit } \\
\text { before treatment experienced at least some clinical } \\
\text { improvement }\end{array}$ \\
\hline Haley et al. ${ }^{(40)}$ & $\begin{array}{c}\text { Prospective case } \\
\text { series }\end{array}$ & $\begin{array}{c}\text { Compare the efficacy } \\
\text { and cost effectiveness } \\
\text { of EBRT versus SRS }\end{array}$ & $\begin{array}{l}44 \text { (22 received EBRT } \\
\text { and } 22 \text { SRS) }\end{array}$ & & $\begin{array}{l}\text { EBRT cost } 29-71 \% \text { of the SRS treatment, had more acute } \\
\text { toxicity (but self-limited and with low grade), and more of } \\
\text { them need further intervention (surgery/kyphoplasty) } \\
\text { No late complication in either groups } \\
\text { Similar pain relief }\end{array}$ \\
\hline
\end{tabular}

\section{Indications}

Primary radiosurgery for treatment of epidural spinal metastases was reported in 13 studies, including spinal sarcomas. These studies were classified as level II and level III of evidence ${ }^{(10-22)}$. Two studies reported the efficacy and safety of radiosurgery in tumors with known low response to CER, and radioresistant tumors such as melanoma and renal cell carcinoma ${ }^{(23,24)}$.

SRS as salvage treatment, after failure of previously irradiated epidural spinal metastases with CER, was reported in 15 studies $^{(10-12,14,15,19,25-33)}$ (levels II and III).

The use of spinal radiosurgery for treatment of intradural extramedullary and intramedullary spinal metastases and for epidural compression of myeloma was reported in one study each (level III) ${ }^{(34,35)}$.
Radiosurgery following surgery for decompression and stabilization was reported in three studies (level III) ${ }^{(33,36-37)}$.

\section{Dose intensity and number of fractions}

The dose obtained in the studies for epidural metastases ranged from 8 to $30 \mathrm{~Gy}$ (different low doses were used for intradural tumors) $)^{(12)}$, in one to five fractions. Moulding et al. considered that when lower doses were used (18 to $21 \mathrm{~Gy})$, worse local control was obtained compared to higher doses (18-24Gy). Some authors administered the same doses for all patients ${ }^{(18)}$, whereas others reported that factors such as prior conventional radiotherapy, distance of the epidural metastases from the $\operatorname{cord}^{(29)}$, 
and tumor histology ${ }^{(23)}$, among others, were used to individualize the dose. It also should be mentioned that lower doses were used for intradural and intramedullar metastases as well as for salvage treatment after prior conventional radiotherapy, in order to avoid late radiation myelopathy.

\section{Pain control}

Although the lack of standardized outcome measurements in the reviewed studies precluded deep or statistical analyses, pain was substantially controlled (up to 90\%) in many series, suggesting good efficacy in decreasing pain symptoms $^{(10-12,14,16,19,23,24,29,33,35,38,39)}$. A potential advantage of SRS is the time required to obtain some pain relief, as early as 24 hours, probably quicker than $\mathrm{CER}^{(21)}$. However, it is important to emphasize that the methods utilized for pain evaluation in different papers were very heterogeneous, which precluded a uniform analysis (verbal analysis scale, and subjective measurements of pain relief, among others). In some tumors considered radioresistant to CER, pain control was achieved in $96 \%$ of the patients with melanomas and in $89 \%$ of the patients with renal cell carcinoma ${ }^{(23,24)}$. However, we also observed that low rates of pain control $(43 \%)$ were obtained in a series using a lower dose of SRS ${ }^{(11)}$.

\section{Toxicity}

Radiation-related toxicity was mild in almost all series, with rare reports of severe toxicity ${ }^{(10,12-13,29,31)}$. Transient radiculitis was reported in 2 of 31 patients of the Benzil et al. series ${ }^{(12)}$. Three patients of 41 in the cases published by Martin et al. presented with toxicity grade $3^{(19)}$. These rarely reported cases of moderate toxicity in all the cases reviewed suggested that SRS is a safe procedure.

\section{Local disease control}

The efficacy of spinal radiosurgery in local disease control was assessed clinically and radiologically in some studies. Chang et al. reported radiological control of spinal metastases in $90 \%$ of the patients at 6 months and in $80 \%$ at 12 months, similar to Garg et al., who obtained an imaging control rate of $88 \%$ of the patients after 18 months of follow-up ${ }^{(15,17)}$. Sheehan et al. ${ }^{(33)}$ also reported decreased or stable tumor volume in $82 \%$ of the patients treated (mean of 12.7 months of follow-up). Some papers also reported improvement in neurological deficits after treatment with SRS ${ }^{(16,25,39)}$.
Regarding tumor recurrence, some interesting information was obtained: Koyfman et al. reported that the presence of paraspinal disease at the time of CER (prior to SRS) was associated with high rates of local recurrence $(p=0.02)^{(28)}$. A dose lower than $16 \mathrm{~Gy}$ was also associated with local recurrence $(\mathrm{p}=0.14)$. Regarding the radiation target, Klish et al. evaluated a prospective series of 58 patients with good disease control and no damage to healthy tissues, and concluded that irradiation one or two levels above or below the involved segment is not necessary when SRS is performed ${ }^{(27)}$.

\section{Cost-effectiveness of CER versus SRS}

In one prospective case series, Haley et al. ${ }^{(40)}$ compared the efficacy/cost- effectiveness of CER versus SRS. At 1-month follow-up, they reported that both methods have similar outcomes regarding pain relief, but CER costs about 29 to $71 \%$ less than the SRS treatment. However, CER has a high rate of acute toxicity (although it was self-limited and with low intensity) and required more interventional procedures (surgery, kyphoplasty etc.) at the level of the treated spinal. Neither method had late complications.

\section{DISCUSSION}

The ideal treatment of spinal metastases would be one that obtains cure without adding excessive morbidity. However, since no treatment has been proven to increase life expectancy, the main objectives of treatment remain local disease control, pain relief, reestablishment of a normal neurological status, and avoidance of spinal deformity/instability. Surgery is reserved for an unstable spine, to obtain tissue samples, for pain relief in cases of instability, or for urgent decompression. Regardless of the indication for initial surgical treatment, almost all patients should be referred to radiation therapy treatment.

As shown in our data, SRS was efficient in controlling pain in many series ${ }^{(10-12,14,16,19,23,24,29,33,34,38,39)}$. Most of the studies showed pain control of up to $90 \%$, even for radioresistant tumors such as melanomas and renal cell carcinoma. Since a cure is not possible, the palliative effect of SRS in pain control has been one of the main reasons for radiation treatment in these patients.

Clinical stability and radiological disease control were obtained in up to $95 \%$ of the series ${ }^{(15,17,33)}$. The efficacy of local control decreased with time, but most of the patients died from progression of the systemic disease before local recurrence ${ }^{(37)}$. The presence of paraspinal disease increases local recurrence probably 
because it is associated with late stages of cancer and a more systemic disease. This radiological control obtained with SRS can decrease or stabilize tumor volume, improving the neurological status in some series ${ }^{(16,35,39)}$. All these features confirm SRS as an efficient method for treating spinal metastases regarding local control.

Ideally, an efficient method that improves the patient's symptoms should not carry high morbidity rates. In this context, the studies assessed in our review with more than 2,000 patients did not demonstrate severe toxicity related to SRS. Moreover, when reported, toxicity was mild and self-limited, attesting to the safety of SRS in treatment of spinal cancer ${ }^{(10,12,13,29,32)}$.

Since safety and efficacy are warranted, the oncology group needs to individualize the patient's treatment and decide between SRS and CER. Although both methods require certainty of the diagnosis, SRS has its best indications for patients with more restricted spinal/ paraspinal metastases (involving one to two spinal segments); on the other hand, CER is more efficient for multiple level spinal involvement ${ }^{(34)}$. As stated before, although costs favor the indication of $\mathrm{CER}^{(40)}$, this assertion does not really reflect the truth, especially for patients who live far from radiotherapy centers, as SRS requires less fractions ( 1 to 5 ) compared to CER ( 8 to 20 daily fractions).

Although many questions are not yet solved, SRS has proven to be an efficient and safe alternative to CER for treating patients with restricted spinal metastases. The best indication for using SRS as a primary treatment of spinal metastases is likely for disease restricted to one or two levels of a known poor responder to CER, such as renal cell carcinoma and melanomas. Another potentially good clinical scenario for SRS is in cases in which CER has failed to achieve local disease control.

\section{CONCLUSION}

SRS can be used for the treatment of epidural spinal metastases (level II of evidence) and spinal myeloma (level III), especially in tumors known to be poor responders to CER (such as melanoma or renal cell carcinoma) (level III). SRS is also indicated for salvage treatment when CER fails to control disease (level II), as reirradiation of patients without any other potential treatment available. There is some evidence (level III) of the efficacy and safety of SRS in the treatment of intradural extramedullary and intramedullary spinal metastases. The same is true for its use after surgical procedures for canal decompression and stabilization (level III). Further prospective studies are necessary to compare the potential benefits of SRS over CER.

\section{REFERENCES}

1. Böhm P, Huber J. The surgical treatment of bony metastases of the spine and limbs. J Bone Joint Surg Br. 2002;84(4):521-9.

2. Wong DA, Fornasier VL, MacNab I. Spinal Metastases: the obvious, the occult and the impostors. Spine (Phila Pa 1976). 1990;15(1):1-4.

3. Perrin $\mathrm{RG}$, Livingston $\mathrm{KE}$, Aarabi $\mathrm{B}$. Intradural extra-medullary spinal metastasis. A report of 10 cases. J Neurosurg. 1982;56(6):835-7.

4. Klimo P Jr, Schmidt MH. Surgical management of spinal metastases. Oncologist. 2004;9(2):188-96.

5. Eastley N, Newey M, Ashford RU. Skeletal metastases - the role of the orthopaedic and spinal surgeon. Surg Oncol. 2012;21(3):216-22.

6. Finn MA, Vrionis FD, Schmidt MH. Spinal radiosurgery for metastatic disease of the spine. Cancer Control. 2007;14(4):405-11.

7. Tokuhashi Y, Matsuzaki H, Toriyama S, Kawano H, Ohsaka S. Scoring system for the preoperative evaluation of metastatic spine tumor prognosis. Spine (Phila Pa 1976). 1990;15(11):1110-3.

8. Tomita K, Kawahara N, Kobayashi T, Yoshida A, Murakami H, Akamaru T. Surgical strategy for spinal metastases. Spine (Phila Pa 1976). 2001;26(3): 298-306.

9. Wright JG, Swiontkowski MF, Heckman JD. Introducing levels of evidence to the journal. J Bone Joint Surg Am. 2003;85-A(1):1-3.

10. Ahmed KA, Stauder MC, Miller RC, Bauer HJ, Rose PS, Olivier KR, et al. Stereotactic body radiation therapy in spinal metastases. Int J Radiat Oncol Biol Phys. 2012;82(5):e803-9.

11. Amdur RJ, Bennett J, Olivier K, Wallace A, Morris CG, Liu C, et al. A prospective, phase ii study demonstrating the potential value and limitation of radiosurgery for spine metastases. Am J Clin Oncol. 2009;32(5):515-20.

12. Benzil DL, Saboori M, Mogilner AY, Rocchio R, Moorthy CR. Safety and efficacy of stereotactic radiosurgery for tumors of the spine. J Neurosurg. 2004;101 Suppl 3:413-8.

13. Chang EL, Shiu AS, Mendel E, Mathews LA, Mahajan A, Allen PK, et al. Phase I/II study of stereotactic body radiotherapy for spinal metastasis and its pattern of failure. J Neurosurg Spine. 2007;7(2):151-60.

14. Chang UK, Youn SM, Park SQ, Rhee CH. Clinical results of cyberknife(r) radiosurgery for spinal metastases. J Korean Neurosurg Soc. 2009;46(6): 538-44.

15. Chang UK, Cho WI, Kim MS, Cho CK, Lee DH, Rhee CH. Local tumor control after retreatment of spinal metastasis using stereotactic body radiotherapy: comparison with initial treatment group. Acta Oncol. 2012;51 (5):589-95.

16. De Salles AA, Pedroso AG, Medin P, Agazaryan N, Solberg T, Cabatan-Awang C, et al. Spinal lesions treated with Novalis-shaped beam intensity-modulated radiosurgery and stereotactic radiotherapy. J Neurosurg. 2004;101(3):435-40.

17. Garg AK, Shiu AS, Yang J, Wang XS, Allen P, Brown BW, et al. Phase 1/2 trial of single-session stereotactic body radiotherapy for previously unirradiated spinal metastases. Cancer. 2012;118(20):5069-77.

18. Levine AM, Coleman C, Horasek S. Stereotactic radiosurgery for the treatment of primary sarcomas and sarcoma metastases of the spine. Neurosurgery. 2009;64(2 Suppl):A54-9.

19. Martin AG, Cowley IR, Taylor BA, Cassoni AM, Landau DB, Plowman PN (Stereotactic) radiosurgery XIX: spinal radiosurgery-two-year experience in a UK centre. Br J Neurosurg. 2012;26(1):53-8.

20. Patel VB, Wegner RE, Heron DE, Flickinger JC, Gerszten P, Burton SA Comparison of whole versus partial vertebral body stereotactic body radiation therapy for spinal metastases. Technol Cancer Res Treat. 2012;11(2):105-15.

21. Ryu S, Rock J, Rosenblum M, Kim JH. Patterns of failure after single-dose radiosurgery for spinal metastasis. J Neurosurg. 2004;101 Suppl 3:402-5.

22. Wang XS, Rhines LD, Shiu AS, Yang JN, Selek U, Gning I, et al. Stereotactic body radiation therapy for management of spinal metastases in patients without spinal cord compression: a phase 1-2 trial. Lancet Oncol. 2012; 13(4):395-402.

23. Gerszten PC, Burton SA, Ozhasoglu C, Vogel WJ, Welch WC, Baar J, et al. 
Stereotactic radiosurgery for spinal metastases from renal cell carcinoma. J Neurosurg Spine. 2005;3(4):288-95.

24. Gerszten PC, Burton SA, Quinn AE, Agarwala SS, Kirkwood JM. Radiosurgery for the treatment of spinal melanoma metastases. Stereotact Funct Neurosurg. 2005;83(5-6):213-21.

25. Choi CY, Adler JR, Gibbs IC, Chang SD, Jackson PS, Minn AY, et al. Stereotactic radiosurgery for treatment of spinal metastases recurring in close proximity to previously irradiated spinal cord. Int J Radiat Oncol Biol Phys. 2010;78(2):499-506

26. Gagnon GJ, Henderson FC, Gehan EA, Sanford D, Collins BT, Moulds JC, et al. Cyberknife radiosurgery for breast cancer spine metastases: a matched-pair analysis. Cancer. 2007;110(8):1796-802.

27. Klish DS, Grossman P, Allen PK, Rhines LD, Chang EL. Irradiation of spinal metastases: should we continue to include one uninvolved vertebral body above and below in the radiation field? Int J Radiat Oncol Biol Phys. 2011; $81(5): 1495-559$.

28. Koyfman SA, Djemil T, Burdick MJ, Woody N, Balagamwala EH, Reddy CA, et al. Marginal recurrence requiring salvage radiotherapy after stereotactic body radiotherapy for spinal metastases. Int J Radiat Oncol Biol Phys. 2012; 83(1):297-302.

29. Mahadevan A, Floyd S, Wong E, Jeyapalan S, Groff M, Kasper E. Stereotactic body radiotherapy reirradiation for recurrent epidural spinal metastases. Int J Radiat Oncol Biol Phys. 2011;81 (5):1500-5.

30. Nikolajek K, Kufeld M, Muacevic A, Wowra B, Niyazi M, Ganswindt U. Spinal radiosurgery--efficacy and safety after prior conventional radiotherapy. Radiat Oncol. 2011:6:173.

31. Sahgal A, Ames C, Chou D, Ma L, Huang K, Xu W, et al. Stereotactic body radiotherapy is effective salvage therapy for patients with prior radiation of spinal metastases. Int J Radiat Oncol Biol Phys. 2009;74(3):723-31.

32. Sahgal A, Ma L, Weinberg V, Gibbs IC, Chao S, Chang UK, et al. Reirradiation human spinal cord tolerance for stereotactic body radiotherapy. Int J Radiat Oncol Biol Phys. 2012;82(1):107-16.

33. Sheehan JP, Shaffrey Cl, Schlesinger D, Williams BJ, Arlet V, Larner J. Radiosurgery in the treatment of spinal metastases: tumor control, survival, and quality of life after helical tomotherapy. Neurosurgery. 2009;65(6):1052-61.

34. Shin DA, Huh R, Chung SS, Rock J, Ryu S. Stereotactic spine radiosurgery for intradural and intramedullary metastasis. Neurosurg Focus. 2009;27(6):E10.

35. Jin R, Rock J, Jin JY, Janakiraman N, Kim JH, Movsas B, et al. Single fraction spine radiosurgery for myeloma epidural spinal cord compression. J Exp Ther Oncol. 2009;8(1):35-41.

36. Massicotte E, Foote M, Reddy R, Sahgal A. Minimal access spine surgery (MASS) for decompression and stabilization performed as an out-patient procedure for metastatic spinal tumours followed by spine stereotactic body radiotherapy (SBRT): first report of technique and preliminary outcomes. Technol Cancer Res Treat. 2012;11(1):15-25.

37. Moulding HD, Elder JB, Lis E, Lovelock DM, Zhang Z, Yamada $Y$, et al Local disease control after decompressive surgery and adjuvant high-dose single-fraction radiosurgery for spine metastases. J Neurosurg Spine. 2010; 13(1):87-93.

38. Garg AK, Wang XS, Shiu AS, Allen P, Yang J, McAleer MF, et al. Prospective evaluation of spinal reirradiation by using stereotactic body radiation therapy: The University of Texas MD Anderson Cancer Center experience. Cancer. 2011;117(15):3509-16.

39. Gerszten PC, Burton SA, Ozhasoglu C, Welch WC. Radiosurgery for spinal metastases: clinical experience in 500 cases from a single institution. Spine. 2007;32(2):193-9.

40. Haley ML, Gerszten PC, Heron DE, Chang YF, Atteberry DS, Burton SA Efficacy and cost-effectiveness analysis of external beam and stereotactic body radiation therapy in the treatment of spine metastases: a matched-pair analysis. J Neurosurg Spine. 2011;14(4):537-42. 